\title{
Effects of saffron extract and crocin on anthropometrical, nutritional and lipid profile parameters of rats fed a high fat diet
}

\begin{abstract}
Overweight and obesity are the most common nutritional disorders in the world. The aim of this study was to evaluate anti-obesity effects of ethanolic extracts of saffron and crocin in comparison to orlistat in animal model. Male Sprague Dawley (SD) rats were fed high-fat diet (HFD) for 12 weeks to induce obesity. The saffron extracts (40 and $80 \mathrm{mg} / \mathrm{kg}$ ), crocin (40 and $80 \mathrm{mg} / \mathrm{kg}$ ) and orlistat $(20 \mathrm{mg} / \mathrm{kg}$ ) were fed to rats by mixing with high fat diet (HFD) for 8 weeks. Changes in anthropometrical, nutritional and lipid profile parameters were measured. The saffron extract significantly decreased food consumption in obese rats. Crocin $(80 \mathrm{mg} / \mathrm{kg})$ showed a significant decrease on rate of body weight gain, total fat pad and weight ratio of epididymal fat to body. Furthermore, crocin $(80 \mathrm{mg} / \mathrm{kg})$ significantly reduced plasma levels of triacylglycerol (TG) and total cholesterol (TC) while saffron extract (40 mg/kg) showed major improvement in low-density lipoprotein to high-density lipoprotein (LDL/HDL) level as atherogenic index. These findings demonstrated the potential anti-obesity benefits of saffron extract and crocin in preclinical study.
\end{abstract}

Keyword: Saffron; Crocin; Obesity; Weight loss; High-fat diet; Rat 